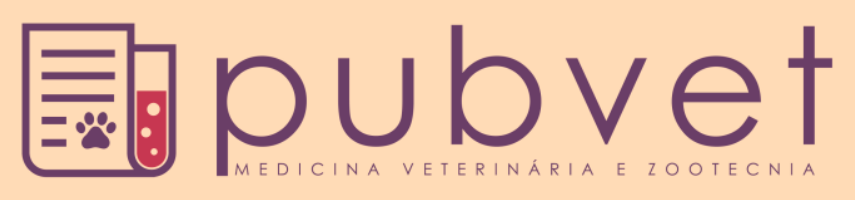

https://doi.org/10.22256/pubvet.v12n6a109.1-6

\title{
Análise físico-química de polpas de acerola (Malpighia glabra L.) artesanais e industriais congeladas
}

\author{
Jaqueline Freitas do Nascimento ${ }^{1} \odot$, Bruno dos Santos Barroso ${ }^{2}$, Ediluci do Socorro \\ Leôncio $\operatorname{Tostes}^{3}{ }^{\circ}$, Anne do Socorro Santos da Silva ${ }^{4}$, Antonio Carlos Souza da Silva \\ Júnior $^{* * \bullet}$ \\ ${ }^{1}$ Acadêmica de Farmácia, Instituto Macapaense de Ensino Superior-IMMES. Macapá-AP Brasil. E-mail: jackllinefn@hotmail.com \\ ${ }^{2}$ Acadêmico de Nutrição, Instituto Macapaense de Ensino Superior-IMMES. Macapá-AP Brasil. E-mail: $\underline{\text { sbruno2016@outlook.com }}$ \\ ${ }^{3}$ Pesquisadora do Instituto de Pesquisas Científicas e Tecnológicas do Estado do Amapá-IEPA, Núcleo de Ciência e Tecnologia de Alimentos. \\ Macapá-AP Brasil. E-mail: ediluci.tostes@hotmail.com \\ ${ }^{4}$ Pesquisadora do Instituto de Pesquisas Científicas e Tecnológicas do Estado do Amapá-IEPA, Núcleo de Ciência e Tecnologia de Alimentos. \\ Macapá-AP Brasil. E-mail: annedosocorro@hotmail.com \\ ${ }^{5}$ Pesquisador do Instituto de Pesquisas Científicas e Tecnológicas do Estado do Amapá-IEPA, Núcleo de Ciência e Tecnologia de Alimentos. \\ Macapá-AP Brasil. E-mail: jr_bio2005@yahoo.com.br *Autor para correspondência.
}

RESUMO. O processamento de frutas para obtenção de polpas é uma atividade agroindustrial importante, na medida em que agrega valor econômico à fruta, evitando desperdícios e minimizando perdas que podem ocorrer durante a comercialização do produto in natura. A polpa de fruta pode substituir a fruta in natura no preparo de sucos, néctares, doces, geleias, sorvetes, apresentando a vantagem de ser encontrada também no período de entressafra dessas frutas. Desta forma, o objetivo deste trabalho é analisar os parâmetros físico-químicos das polpas de acerola industrialmente e artesanalmente fabricadas e comercializadas na cidade de Macapá-AP. No estudo foram utilizadas 6 amostras, sendo que 3 eram polpas produzidas artesanalmente e as 3 restantes polpas produzidas industrialmente onde foram feitas análises físico-químicas realizadas em triplicata, com parâmetros como pH, Sólidos Solúveis Totais (SST), Acidez Total Titulável (ATT), Umidade, Proteína, Lipídios, Fibra e Ácido Ascórbico (Vitamina C). Dos dados obtidos, os parâmetros como SST das polpas de acerola industrializadas tiveram média de $5,58 \pm 0,1^{\circ} \mathrm{B}$, já as polpas produzidas artesanalmente média de $5,08 \pm 1,2^{\circ} \mathrm{B}$. Tendo como Padrão de Identidade e Qualidade (PIQ), para SST o valor mínimo de $5,50^{\circ} \mathrm{B}$, estando dessa forma as polpas artesanais abaixo do padrão estabelecido. Para o ácido ascórbico nas polpas industriais a média foi de $1080,11 \pm 21,02 \mathrm{mg} / 100 \mathrm{~g}$. Enquanto nas polpas artesanais a média encontrada foi de $633,04 \pm 118,77 \mathrm{mg} / 100 \mathrm{~g}$, dessa forma, apenas as polpas industriais estavam dentro do que preconiza a legislação que determina mínimo de $800 \mathrm{mg} / 100 \mathrm{~g}$ de ácido ascórbico. $\mathrm{O}$ pH para ambas as polpas atendiam a legislação que preconiza $\mathrm{pH}$ acima de 2,80. Quanto aos parâmetros de proteínas, lipídios, fibras e umidade se enquadrando na natureza geral da maioria dos produtos vegetais, exceto oleaginosas. Considerando os achados desta pesquisa, as polpas industrializadas apresentaram valores mais compatíveis com a legislação e com a literatura do que as de produção artesanal, acredita-se que seja decorrente do processo de industrialização que influencia o seu estado de conservação.

Palavras-chave: Frutas tropicais, PIQ, Vitamina C

\section{Physico-chemical analysis of pulps of western indian cherry (Malpighia glabra L.) handmade and industrial frozen}

ABSTRACT. The processing of fruit to obtain pulps is an important agroindustrial activity, since it adds economic value to the fruit, avoiding wastes and minimizing losses 
that can occur during the commercialization of the product in natura. Fruit pulp can replace fresh fruit in the preparation of juices, nectars, jams, jellies, ice creams, with the advantage of being found also in the off-season period of these fruits. In this way, the objective of this work is to analyze the physical-chemical parameters of the acerola pulp industrially and artisanally manufactured and commercialized in the city of Macapá-AP. In the study, 6 samples were used, 3 of which were hand-crafted pulps and the remaining 3 industrialproduced pulps were made in triplicate, with parameters such as $\mathrm{pH}$, Total Soluble Solids (TSS), Titratable Total Acidity, Moisture, Protein, Lipids, Fiber and Ascorbic Acid (Vitamin C). From the data obtained, the parameters as SST of the industrialized acerola pulp had a mean of $5.58 \pm 0.1^{\circ} \mathrm{B}$, while the handmade pulp produced average of $5.08 \pm$ $1.2^{\circ} \mathrm{B}$. Having as Identity and Quality Standard (SIQ), for SST the minimum value of 5.50 ${ }^{\circ} \mathrm{B}$, thus being the handmade pulps below the established standard. For ascorbic acid in industrial pulps the mean was $1080.11 \pm 21.02 \mathrm{mg} / 100 \mathrm{~g}$. While in the traditional pulps the average found was $633.04 \pm 118.77 \mathrm{mg} / 100 \mathrm{~g}$, in this way, only the industrial pulps were within the one recommended by the legislation that determines a minimum of $800 \mathrm{mg}$ / $100 \mathrm{~g}$ of ascorbic acid. The $\mathrm{pH}$ for both pulps complied with the legislation that recommends $\mathrm{pH}$ above 2.80 . Regarding the parameters of proteins, lipids, fibers and moisture, they fit into the general nature of most vegetable products, except oilseeds. Considering the findings of this research, the industrialized pulps presented values more compatible with the legislation and with the literature than those of artisanal production, it is believed that it is due to the process of industrialization that influences its state of conservation.

Keywords: Tropical fruts, SIQ, Vitamin C

\section{Análisis físico-químico de pulpa de acerola (Malpighia glabra L.) artesanales $e$ industriales congelados}

RESUMEN. El procesamiento de frutas para la obtención de pulpas es una actividad agroindustrial importante, en la medida en que agrega valor económico a la fruta, evitando desperdicios y minimizando pérdidas que pueden ocurrir durante la comercialización del producto in natura. La pulpa de fruta puede sustituir la fruta in natura en la preparación de jugos, néctares, dulces, jaleas, helados, presentando la ventaja de ser encontrada también en la época de baja oferta de esas frutas. De esta forma, el objetivo de este trabajo es analizar los parámetros físico-químicos de las pulpas de acerola industrialmente y artesanalmente fabricadas y comercializadas en la ciudad de Macapá-AP. En el estudio se utilizaron 6 muestras, siendo que 3 eran pulpas producidas artesanalmente y las 3 restantes pulpas producidas industrialmente donde se realizaron análisis físico-químicos realizados en triplicada, con parámetros como pH, Sólidos Solubles Totales (SST), Acidez Total Titulable (ATT), Humedad, Proteína, Lipídios, Fibra y Ácido Ascórbico (Vitamina C). De los datos obtenidos, los parámetros como SST de las pulpas de acerola industrializadas tuvieron media de $5,58 \pm 0,1^{\circ} \mathrm{B}$, ya las pulpas producidas artesanalmente promedio de $5,08 \pm 1,2^{\circ} \mathrm{B}$. Con un valor mínimo de $5,50^{\circ} \mathrm{B}$, de acuerdo con la norma de identidad y calidad (PIQ), para SST el valor mínimo de $5,50^{\circ} \mathrm{B}$, estando las pulpas artesanales por debajo del estándar establecido. Para el ácido ascórbico en las pulpas industriales la media fue de 1080,11 $\pm 21,02 \mathrm{mg} / 100 \mathrm{~g}$. Mientras que en las pulpas artesanales la media encontrada fue de $633,04 \pm 118,77 \mathrm{mg} / 100 \mathrm{~g}$, de esa forma, sólo las pulpas industriales estaban dentro de lo que preconiza la legislación que determina mínimo de $800 \mathrm{mg} / 100 \mathrm{~g}$ de ácido ascórbico. El pH para ambas pulpas atendía a la legislación que preconiza el pH por encima de 2,80. En cuanto a los parámetros de proteínas, lípidos, fibras y humedad se encuadran en la naturaleza general de la mayoría de los productos vegetales, excepto oleaginosas. Considerando los hallazgos de esta investigación, las pulpas industrializadas presentaron valores más compatibles con la legislación y con la literatura que las de producción artesanal, se cree que es consecuencia del proceso de industrialización que influye en su estado de conservación.

Palabras clave: Frutas tropicales, PIQ, Vitamina C 


\section{Introdução}

Segundo Brasil (2000), a polpa de fruta é resultante da parte comestível do fruto que é produzida por meio de um processo tecnológico adequado obtido de frutos polposos, sendo definida como um produto de teor mínimo de sólidos totais, não fermentado, não diluído e não concentrado.

$\mathrm{O}$ processo mais utilizado na elaboração e conservação de polpas de frutas é o congelamento, pois a sua aceitação é maior devido a preservação das características sensoriais do fruto (Salgado et al., 1999). Existem também outros processos que podem ser empregados como: conservação por aditivo químico e a pasteurização, mas na maioria das empresas o processo utilizado para conservação de polpas é apenas o congelamento (Santos et al., 2004).

Os parâmetros que são importantes para a regulamentação do produto e a investigação de alterações realizadas no decorrer do armazenamento e/ou processamento são os sólidos solúveis, vitamina $\mathrm{C}, \mathrm{pH}$, açucares redutores e totais e acidez titulável, com o propósito de manutenção do controle de qualidade de polpas de fruta (Benevides et al., 2008). O teor de sólidos solúveis é uma variação do teor de açúcar especifico ao tipo de fruto e pode diversificar tanto em virtude da adição ocasional de água no decorrer do processamento, como em razão de fatores climáticos. A acidez e o pH são fatores importantes para determinar o processo de conservação de polpas (Lira Júnior et al., 2005; Santos et al., 2004).

De acordo com Danieli et al. (2009), a vitamina $\mathrm{C}$ é oxidada de forma acelerada quando exposta ao ar, sendo fotolábil, termolábil e hidrossolúvel. Também é usada como um marcador de qualidade nutricional de produtos derivados de frutas pois ela possui a capacidade de se degradar durante o armazenamento e processamento através de sua alta sensibilidade.

Diante disso, o objetivo deste trabalho é analisar os parâmetros físico-químicos das polpas de acerola industrialmente e artesanalmente fabricadas e comercializadas na cidade de Macapá-AP.

\section{Material e Métodos}

\section{Aquisição da matéria prima}

Foram utilizadas 6 amostras, sendo que 3 eram polpas produzidas artesanalmente e as 3 restantes polpas produzidas industrialmente. As polpas de acerola congeladas de produção artesanal foram adquiridas diretamente dos vendedores em feira livre e as de produção industrial em supermercado da cidade de Macapá. Foram transportadas em caixas de material isotérmico para o laboratório de análises físico-químicas no Núcleo de Ciência e Tecnologia de Alimentos do Instituto de Pesquisas Científicas e Tecnológicas do Estado do Amapá IEPA.

Para as análises físico-químicas realizadas em triplicata, os parâmetros como pH, Sólidos Solúveis Totais (SST), Acidez Total Titulável (ATT), Umidade, Proteína, Lipídios, Fibra e Ácido Ascórbico (Vitamina C), foram utilizadas as metodologias do IAL (2008) e da AOAC (2000), que se tratam das metodologias padrões para análises físico-químicas de alimentos.

As análises dos resultados obtidos foram realizadas pela utilização de estatística descritiva com o auxílio do programa BioEstat 5.0 e comparação com os trabalhos já realizados e com os parâmetros da legislação vigente.

\section{Resultados e Discussão}

Na Tabela 1 estão demonstrados os resultados obtidos através da determinação físico-química das polpas industrializadas e de produção artesanal da acerola.

Tabela 1. Resultados das análises físico-químicas das polpas de acerola de produção artesanal e industrial.

\begin{tabular}{lccc}
\hline ANÁLISE & ACE IND & ACE ART & PIQ \\
\hline ATT $(\% \mathrm{v} / \mathrm{p})$ & $20,13 \pm 3,2$ & $20,24 \pm 6,2$ & - \\
SST $\left({ }^{\circ} \mathrm{B}\right)$ & $5,58 \pm 0,1$ & $5,08 \pm 1,2$ & 5,50 \\
pH & $3,35 \pm 0,1$ & $3,63 \pm 0,2$ & 2,80 \\
Lipídeos $(\%)$ & $0,99 \pm 0,8$ & $0 \pm 0$ & - \\
Fibras $(\%)$ & $0,53 \pm 0,4$ & $0 \pm 0$ & - \\
Umidade $(\%)$ & $93,61 \pm 0,4$ & $95,67 \pm 1,6$ & - \\
Proteínas $(\%)$ & $2,71 \pm 1$ & $2,25 \pm 0,6$ & - \\
AA (mg/100g) & $1080,11 \pm 21,02$ & $633,04 \pm 118,77$ & 800
\end{tabular}

ACE IND: Polpa de Acerola de produção industrial; ACE ART: Polpa de Acerola de produção artesanal; ATT: Acidez Total Titulável; SST: Sólidos Solúveis Totais; AA: Ácido Ascórbico; PIQ: Padrão de Identidade e Qualidade (Brasil, 2000).

As polpas de acerola industrializadas apresentaram variação de ATT entre 16,70 e $24,43 \%$, tendo como média $20,13 \pm 3,2 \%$, enquanto que a polpa produzida artesanalmente variou para 
o mesmo parâmetro entre 12,82 e $28 \%$, tendo como média $20,24 \pm 6,2 \%$. Resultados semelhantes foram encontrados no trabalho de Bueno et al. (2002), onde foi relatada ATT de 21,0\% em polpas de acerola de um supermercado de grande porte da cidade de São José do Rio Preto - SP.

A acidez é um parâmetro relevante na avaliação do estado de conservação de um produto alimentício (Castro et al., 2015). As concentrações dos íons de hidrogênio são alteradas devido ao processo de decomposição do alimento realizadas tanto por fermentação, hidrólise ou oxidação tendo como consequência a sua acidez (Aroucha et al., 2010). Com o processo de maturação do fruto, a acidez geralmente é reduzida, vindo a influenciar o odor, sabor, cor, manutenção de qualidade e estabilidade (Pereira et al., 2006).

Quando avaliados os SST, as polpas de acerola industrializadas tiveram variação entre 5,5 a 5,75 com média de $5,58 \pm 0,1^{\circ} \mathrm{B}$, já as polpas produzidas artesanalmente SST variaram de 3,50 a $6,25^{\circ} \mathrm{B}$, tendo como média $5,08 \pm 1,2^{\circ} \mathrm{B}$. De acordo com o Padrão de Identidade e Qualidade (PIQ) segundo a Instrução Normativa $n^{\circ} 1$ do Ministério da Agricultura, Abastecimento e Pecuária (Brasil, 2000), SST para a polpa de acerola é de $5,50^{\circ} \mathrm{B}$, estando dessa forma as polpas artesanais abaixo do padrão estabelecido.

Os sólidos solúveis são empregados como índice de maturidade de alguns frutos e apontam a quantidade de substancias que são encontradas dissolvidas nos sucos, sendo formadas por compostos solúveis em água, tais como: ácidos, pectinas, açúcares, compostos orgânicos, fenólicos e vitaminas (Pereira et al., 2006). A diminuição dos teores de sólidos solúveis do produto final pode variar com diversos fatores, entre eles, características climáticas, solo, variedade do fruto, além da adição de água durante o processamento (Chitarra \& Chitarra, 2005).

No trabalho realizado por Ferreira-Marçal et al. (2013) as polpas de acerola industriais comercializadas no município de Governador Valadares-MG, apresentaram valores de SST acima do recomendado pela legislação. Feitosa et al. (2017), avaliando as características físicoquímicas de polpas de acerola comercializadas em Picos-PI também encontraram valores abaixo do recomendado na legislação vigente.

Os valores para $\mathrm{pH}$ foram ácidos com uma variação de 3,19 a 3,48, tendo como média $3,35 \pm 0,1$ para a polpa de acerola industrial, não tendo diferenças significativas quando comparadas com as polpas artesanais, que tiveram variação de 3,43 a 3,91, tendo como média $3,63 \pm 0,2$. Na legislação vigente (Brasil, 2000) o pH mínimo para a polpa de acerola deve ser 2,80, estando o $\mathrm{pH}$ de todas as amostras avaliadas dentro do que preconiza a legislação.

No trabalho de Castro et al. (2015) e Brasil (2016), foram encontrados valores de $\mathrm{pH}$ acima do valor mínimo permitido bem como deste trabalho. E nas conclusões encontradas em Feitosa et al. (2017) 100\% das amostras de polpas de acerolas também apresentaram valores dentro do determinado pela legislação.

De acordo com Benevides et al. (2008), os valores de $\mathrm{pH}$ baixos são significativos, pois eles podem assegurar a conservação da polpa sem necessitar de tratamento térmico elevado, evitando perda de qualidade nutricional. É importante ressaltar que o pH também é entendido como um atributo de qualidade do produto pela legislação, por propiciar a conservação da polpa, fazendo com que o crescimento de leveduras seja evitado (Lira Júnior et al., 2005).

Para o ácido ascórbico na polpa industrial houve variação de 1051,26 a $1100,74 \mathrm{mg} / 100 \mathrm{~g}$, tendo uma média de $1080,11 \pm 21,02 \mathrm{mg} / 100 \mathrm{~g}$. Na polpa artesanal a variação ocorreu entre 538,37 e $800,53 \mathrm{mg} / 100 \mathrm{~g}$, tendo uma média de $633,04 \pm 118,77 \mathrm{mg} / 100 \mathrm{~g}$. Dessa forma, apenas as polpas industriais estavam dentro do que preconiza a legislação vigente (Brasil, 2000) que determina mínimo de $800 \mathrm{mg} / 100 \mathrm{~g}$ de ácido ascórbico.

Segundo os resultados encontrados por Batista et al. (2013), as polpas de acerola comercializadas no município de Datas-MG apresentaram valores abaixo do mínimo estabelecido, assim como as polpas artesanais avaliadas neste trabalho. Nas conclusões encontradas em Scherer et al. (2008), as polpas de acerola comercializadas CampinasSP, tiveram resultados abaixo do estabelecido pela legislação tal como as polpas mensuradas neste estudo. No trabalho de Brasil (2016), 80\% das marcas analisadas logo após a aquisição das polpas estavam em concordância com o PIQ preconizado pela legislação, bem como os resultados das polpas industriais encontradas neste estudo.

O baixo teor de vitamina $\mathrm{C}$ pode estar associado a deficiências do processo produtivo, como emprego de congelamento lento, falhas na 
seleção da matéria prima e a diluição do produto através da adição de água ou ser atribuído as características da matéria prima como grau de maturação, clima, o cultivo e práticas de cultura (Sebastiany et al., 2009).

A polpa industrial apresentou lipídeos $(0,99 \pm 0,8 \%)$ e fibras brutas $(0,53 \pm 0,4 \%)$, enquanto que na polpa artesanal não foram encontrados valores detectáveis para ambos os parâmetros. Quanto as proteínas e umidade, não foram observadas diferenças significativas entre as polpas industriais e artesanais. No trabalho de Soares et al. (2001), foi encontrado valor de $0,21 \%$ de lipídeos e conteúdo proteico de $1,27 \%$ se enquadrando na natureza geral da maioria dos produtos vegetais, exceto oleaginosas.

As diferenças nos constituintes em polpas de frutas são provenientes do fruto (maturação, solo, estação do ano e sistema de produção) e do manuseio (armazenamento, acondicionamento, processamento e transporte), fazendo com que ocorra mudanças entre as amostras nas variáveis estudadas, podendo demonstrar deficiências no que se refere ao controle de qualidade da matériaprima utilizada no processo produtivo de polpas, seja artesanal ou industrial (Carvalho et al., 2017).

Considerando os achados desta pesquisa, as polpas industrializadas apresentaram valores mais compatíveis com a legislação e com a literatura do que as de produção artesanal. Estas demonstraram melhor qualidade físico-química que as de produção artesanal, acredita-se que seja decorrente do processo de industrialização que influencia o seu estado de conservação. A má qualidade das polpas que são produzidas de forma artesanal, justifica-se provavelmente pelo processamento e manipulação de maneira inadequada não seguindo as condutas exigidas em lei pelo Ministério da Agricultura e Abastecimento (MAPA) na Instrução Normativa $n^{\circ}$ 01/2000 (Brasil, 2000) e/ou Agência Nacional de Vigilância Sanitária (ANVISA) segundo a resolução n²75/2002 (Brasil, 2002).

Vale ressaltar também que as polpas de acerola são derivadas de frutas cítricas que possuem alto teor de vitamina C. O ácido ascórbico é um dos nutrientes mais instáveis, portanto necessita de maior atenção para as condições apropriadas de armazenamento e manipulação. Novamente, as polpas produzidas industrialmente apresentaram níveis consideravelmente maiores que as polpas produzidas artesanalmente, o que ainda sugere que o processo de industrialização propicia maior conservação desta vitamina nestas polpas.

\section{Referências bibliográficas}

AOAC. 2000. Official Methods of Analysis (16th ed.). Arlington, VA: AOAC International.

Aroucha, E. M. M., de Gois, V. A., de Lima Leite, R. H., Santos, M. C. A. \& Souza, M. S. 2010. Acidez em frutas e hortaliças. Revista Verde de Agroecologia e Desenvolvimento Sustentável, 5(2), 01-04.

Batista, A., Oliveira, B., Oliveira, M., Guedes, T., Silva, D. \& Pinto, N. 2013. Parâmetros de qualidade de polpas de frutas congeladas: uma abordagem para produção do agronegócio familiar no Alto Vale do Jequitinhonha. Tecnologia \& Ciência Agropecuária, 7(4), 4954.

Benevides, S. D., Mota Ramos, A., Stringheta, P. C. \& Castro, V. C. 2008. Qualidade da manga e polpa da manga Ubá. Ciência e Tecnologia de Alimentos, 28(3), 571-578 .

Brasil, A. 2016. Avaliação da qualidade físicoquímica de polpas de fruta congeladas comercializadas na cidade de Cuiabá-MT. Revista Brasileira de Fruticultura, Jaboticabal-SP, 38(1), 167-175.

Brasil. 2000. Instrução Normativa $n^{\circ} 01 / 00$, de 07/01/00. Regulamento técnico geral para fixação dos padrões de identidade e qualidade para polpa de fruta. Diário Oficial da República Federativa do Brasil, Brasília, DF, 10 jan.2000. Seção 1, p.54-58.

Brasil. 2002. Agência Nacional de Vigilância Sanitária. Resolução n. 275, de 21 de outubro de 2002. Regulamento Técnico de Procedimentos Operacionais Padronizados aplicados aos Estabelecimentos Produtores / Industrializadores de Alimentos e a Lista de Verificação das Boas Práticas de Fabricação em Estabelecimentos Produtores / Industrializadores de Alimentos. Diário Oficial [da] República do Brasil, Brasília, DF, D.O.U. nº 206, de 23-10-2002, Seção 1, pág. 126.

Carvalho, A. V., de Andrade Mattietto, R. \& Beckman, J. C. 2017. Estudo da estabilidade de polpas de frutas tropicais mistas congeladas utilizadas na formulação de bebidas. Brazilian Journal of Food Technology, 20, e2016023.

Castro, T. M. N., Zamboni, P. V., Dovadoni, S., Neto, A. C. \& Rodrigues, L. J. 2015. 
Parâmetros de qualidade de polpas de frutas congeladas. Revista do Instituto Adolfo Lutz, 74(4), 426-436.

Chitarra, M. I. F. \& Chitarra, A. B. 2005. Póscolheita de frutos e hortaliças: Fisiologia e Manuseio ( $2^{\mathrm{a}}$ ed.). Lavras, MG: Editora UFLA.

Danieli, F., Costa, L., Silva, L. C. d., Hara, A. \& Silva, A. A. d. 2009. Determinação de vitamina $\mathrm{C}$ em amostras de suco de laranja in natura e amostras comerciais de suco de laranja pasteurizado e envasado em embalagem Tetra Pak. Revista do Instituto de Ciencias da Saúde, 27(4), 361-365.

Feitosa, R. C., Sousa, A. C. P., Teixeira, S. A. \& Medeiros, S. R. A. 2017. Avaliação da rotulagem da qualidade microbiológica e físico-química de polpas de frutas comercializadas em Picos-PI. Revista Intertox de Toxicologia, Risco Ambiental e Sociedade, 10(2), 62-72.

Ferreira-Marçal, P. H., Dias-Souza, M. V., Villela, E. G., Luz, L. M., Pereira, M. K., Vale, C. H. B. d. \& Rabelo, F. L. A. 2013. Qualidade físico-química, microbiológica e microscópica de polpas de frutas congeladas comercializadas no município de Governador Valadares, MG. NOV@: Revista Científica, 2(2), 1-10.

IAL, I. A. L. 2008. Normas analíticas do Instituto Adolfo Lutz: métodos químicos e físicos para análises de alimentos (4.ed.). São Paulo, SP, Brasil, 1002 p.

Lira Júnior, J. S., Santos Musser, R., Almeida Melo, E., Sucupira Maciel, M. I., Lederman, I. E. \& Santos, V. F. 2005. Caracterização física e físico-química de frutos de cajá-umbu (Spondias spp.). Ciência e Tecnologia de Alimentos, 25(4), 757-761.

Pereira, J. M. d. A. K., Oliveira, K., Soares, N. d. F. F., Gonçalves, M. P. J. C., Pinto, C. L. O. \&
Fontes, E. A. F. 2006. Avaliação da qualidade físico-química, microbiológica e microscópica de polpas de frutas congeladas comercializadas na cidade de Viçosa-MG. Alimentos e Nutrição Araraquara, 17(4), 437-442.

Salgado, S. M., Guerra, N. B. \& Melo Filho, A. d. 1999. Polpa de fruta congelada: efeito do processamento sobre o conteúdo de fibra alimentar. Revista de Nutrição, 12(3), 303-308.

Santos, F. A. d., Salles, J. R. d. J., Chagas Filho, E. \& Rabelo, R. N. 2004. Análise qualitativa de polpas congeladas de frutas, produzidas pela SUFRUTS, MA. Higiene Alimentar, 18-22.

Scherer, R., Rybka, A. C. P. \& Godoy, H. T. 2008. Determinação simultânea dos ácidos orgânicos tartárico, málico, ascórbico e cítrico em polpas de acerola, açaí e caju e avaliação da estabilidade em sucos de caju. Química Nova, 31(5), 1137-1140.

Sebastiany, E., Moura, E. R., Rêgo, E. R. \& Vital, M. J. S. 2009. Perda de vitamina C durante o armazenamento de polpa de acerola congelada. Boletim do Centro de Pesquisa de Processamento de Alimentos, 27(2), 281-288.

Soares, E. C., Oliveira, G. S. F. d., Maia, G. A., Monteiro, J. C. S., Silva Junior, A. \& Filho, M. d. S. M. d. S. 2001. Desidratação da polpa de acerola (Malpighia emarginata dc) pelo processo. Food Science and Technology, 21(2), 164-170.

Article History:

Received 30 March 2018

Accepted 20 April 2018

Available online 31 May 2018

License information: This is an open-access article distributed under the terms of the Creative Commons Attribution License 4.0, which permits unrestricted use, distribution, and reproduction in any medium, provided the original work is properly cited. 